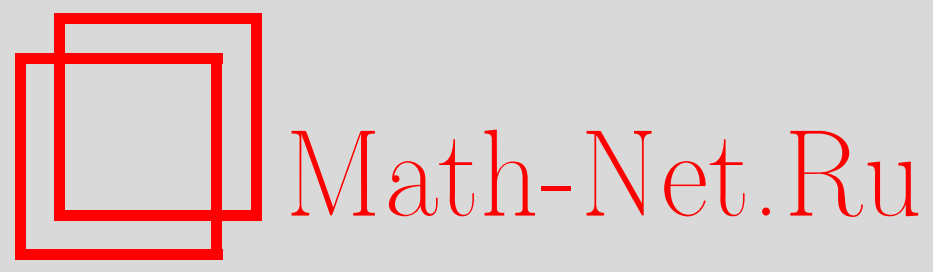

С. Гонсалес, Е. Коусело, В. Т. Марков, А. А. Нечаев, Групповые коды и их неассоциативные обобщения, Дискрет. матем., 2004, том 16, выпуск 1, 146-156

DOI: https://doi.org/10.4213/dm149

Использование Общероссийского математического портала Math-Net.Ru подразумевает, что вы прочитали и согласны с пользовательским соглашением http: //www . mathnet.ru/rus/agreement

Параметры загрузки:

IP : 54.166 .219 .16

26 апреля 2023 г., 18:34:17

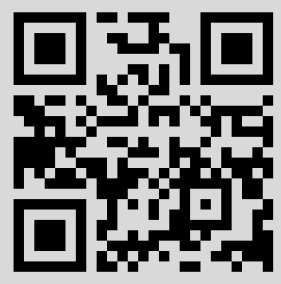


УдК 519.7

\title{
Групповые коды и их неассоциативные обобщения
}

\author{
() 2004 г. С. Гонсалес, Е. Коусело, В. Т. Марков, А. А. Нечаев
}

Дается полное описание (с использованием компьютера) наилучших параметров линейных кодов, соответствующих левым идеалам луповых алгебр $\mathbf{F}_{q} L$ при $q \in\{2,3,4,5\}$ и $|L| \leqslant 7$, а также групповых алгебр $\mathbf{F}_{q} G$ для групп $G$ порядка $|G| \leqslant 12$. Мы выделяем линейно оптимальные коды, коды, удовлетворяюшие условию Варшамова-Гилберта, а также коды, для которых достигается граница Плоткина. Результаты дают основание предположить, что исследование кодов, построенных с помощью неассоциативных и некоммутативных неполупростых алгебр может открыть новые возможности и заслуживает дальнейшего развития.

Работа поддержана Российским фондом фундаментальных исследований, проекты 99-01-00382 и 99-01-00941, и грантами НШ-1910.2003.1 и НШ-2358.2003.9 Президента Российской Федерации для подлержки ведущих научных школ.

В Т. Марков и А. А. Нечаев благодарят Университет Овьедо за гостеприимство.

\section{1. Введение}

В данной статье продолжаются наши исследования кодов, построенных с помощью неассоциативных и некоммутативных алгебраических структур. В первой серии результатов этого направления (см. [1-5]) были описаны различные классы рекурсивных кодов с законами рекурсии, основанными на свойствах $m$-квазигрупп для $\boldsymbol{m} \geqslant 2$.

Другой естественный подход состоит в изучении кодов, построенных с помощью левых идеалов луповых колец $A=R L$, где $R$ - конечное кольцо, а $L$ - конечная группа.

Пусть

$$
L=\left\{g_{1}, \ldots, g_{n}\right\}
$$

- конечная лупа порядка $n, R-$ конечное кольцо, $|R|=q$. Рассмотрим луповое кольцо $A=R L$ и левый идеал $I \leqslant{ }_{A} A$ кольца $A$. Тогда множество $\mathscr{K}=\mathscr{K}(I)$ всех слов $\mathbf{r}=\left(r_{1}, \ldots, r_{n}\right) \in R^{n}$ таких, что $\sum_{i=1}^{n} r_{i} g_{i} \in I$, является левым линейным $n$-кодом над кольцом $R$, который мы будем называть луповым кодом или $L$-кодом. Ясно, что $\mathcal{K}-$ подмодуль модуля ${ }_{R} R^{n}$, и если $C=|\mathscr{K}|$, то $C \leqslant q^{n}$.

Каждый элемент $\mathbf{r} \in \mathcal{K}$ называется кодовым словом. Для любых двух кодовых слов $\mathbf{r}=\left(r_{1}, \ldots, r_{n}\right), \mathbf{r}^{\prime}=\left(r_{1}^{\prime}, \ldots, r_{n}^{\prime}\right)$ вес Хемминга $w_{H}(\mathbf{r})$ и расстояние Хемминга $d\left(\mathbf{r}, \mathbf{r}^{\prime}\right)$ определяются равенствами

$$
\begin{aligned}
w_{H}(\mathbf{r}) & =\left|\left\{i \in\{0,1, \ldots, n-1\}: r_{i} \neq 0\right\}\right|, \\
d\left(\mathbf{r}, \mathbf{r}^{\prime}\right) & =w_{H}\left(\mathbf{r}-\mathbf{r}^{\prime}\right) .
\end{aligned}
$$


Расстояние Хемминга кода $\mathscr{K}$ определяется как число

$$
d=d(\mathscr{K})=\min \{d(\mathbf{u}, \mathbf{v}): \mathbf{u}, \mathbf{v} \in \mathscr{K}, \mathbf{u} \neq \mathbf{v}\}
$$

и ввиду линейности кода, разумеется, удовлетворяет равенству

$$
d=\min \left\{w_{H}(\mathbf{r}): \mathbf{r} \in \mathscr{K} \backslash 0\right\} .
$$

Говорят, что $\mathscr{K}$ является $(n, C, d)_{q}$-кодом или, эквивалентно, $[n, k, d]_{q}$-кодом, где $k=\log _{q} C$ - комбинаторная размерность кода $\mathcal{K}$. Иногда, для краткости, мы будем отождествлять кодовое слово $\mathbf{r}=\left(r_{1}, \ldots, r_{n}\right) \in \mathscr{K}(I)$ с соответствующим элементом $\sum_{i=1}^{n} r_{i} g_{i} \in I$ и говорить, что луповой код содержится в соответствующем луповом кольце.

Известно много фактов о $G$-кодах этого типа, когда $R=\mathrm{F}_{q}-$ конечное поле, $(n, q)=1$ и $G$ - абелева, и в особенности циклическая, группа (классические циклические коды, см. $[8,7])$. В [11-13] приведены некоторые результаты о групповых кодах неабелевых групп, главным образом при условии $(n, q)=1$ полупростоты группового кольца $\mathbf{F}_{q} G$.

Первым шагом в исследовании луповых кодов может быть вычисление параметров всевозможных кодов вида $\mathscr{K}=\mathscr{K}(I)$ для всех левых идеалов $I$ луповых алгебр $\mathbf{F} G$ маленьких порядков и поиск наилучших кодов среди них.

Здесь приводятся результаты вычислений параметров кодов $\mathscr{K}(I)$, где $I$ пробегает множество левых идеалов луповых алгебр $F L$ для полей $F \in\left\{F_{2}, F_{3}, F_{4}, F_{5}\right\}$ и для всех луп $L$ порядков $4, \ldots, 7$. Отдельно мы определяем параметры групповых кодов для групп диэдра $\mathscr{D}_{m}, m=4,5,6$; групшы кватернионов $Q_{8}$; знакопеременной группы $A_{4}$ и всех абелевых групा тех же порядков.

Мы обращаем особое внимание на хорошие коды, то есть коды, параметры которых приближаются к параметрам лучших кодов в следующих смыслах: МДР-коды, линейно оптимальные коды, коды, лежащие на границе Плоткина и удовлетворяющие условию Варшамова-Гилберта. Для этого мы получили, посредством компьютерного перебора, список всех левых идеалов $I$ и, таким образом, список всех возможных луповых кодов $\mathscr{K}=\mathscr{K}(I)$ в упомянутых выше случаях. Мы упорядочили их по высоте (длина максимальной цепочки левых идеалов, начинающейся с нулевого идеала и заканчивающейся данным идеалом), а в случае совпадения высот - по рангу, то есть минимальному числу образующих.

\section{2. Алгоритм перебора}

Приведем краткое описание алгоритма, использованного для поиска всех луповых кодов, то есть всех левых идеалов луповой алгебры заданной лупы, а также для установления вложений одних идеалов в другие.

Алгоритм состоит из двух основных шагов.

Основной шаг 1: Создание списка всех главных левых идеалов.

Для этого реализована следующая процедура.

Шаг 1. Последовательно перебираются элементы $f$ алгебры $A$, начиная, для определенности, с элемента $f=e$. Для исключения повторений рассматриваются только элементы со старшим коэффициентом $1 \in \mathbf{F}_{q}$. 
Цель шагов 2-4 - построить базис главного левого идеала ${ }_{A}(f)$ над полем $\mathbf{F}_{q}$. Для этого используется следующее утверждение.

Предложение 1. Пусть $f \in A=F L-$ произвольный элемент луповой алгебры $F L$. Положим

$$
\mathscr{L}_{0}=0, \quad \mu_{0}=\mathscr{L}_{1}=\langle L(F f)\rangle_{F}, \quad \mathscr{L}_{i+1}=\left\langle L \mathscr{L}_{i}\right\rangle_{F},
$$

и выберем некоторые прямые дополнения $\mu_{i}$ подпространств $\mathscr{L}_{i}$ в $\mathscr{L}_{i+1}$ так, что

$$
\mathscr{L}_{i+1}=\mathscr{L}_{i} \oplus \mu_{i}, \quad i=1,2, \ldots,
$$

где $\langle L X\rangle_{F}$ - линейная оболочка над $F$ множества $\{g x \mid g \in L, x \in X\}$. Тогда

$$
\mathscr{L}_{i+1}=\left\langle L M_{i-1}\right\rangle_{F}+\mathscr{L}_{i}, \quad i=1,2, \ldots
$$

$u$

$$
\bigcup_{i \geqslant 0} \mathscr{L}_{i}={ }_{A}(f)
$$

Действительно, первые равенства выполнены по определению:

$$
\begin{aligned}
\mathscr{L}_{i+1} & =\left\langle L \mathscr{L}_{i}\right\rangle_{F}=\left\langle L\left(\mu_{i-1} \oplus \mathscr{L}_{i-1}\right)\right\rangle_{F} \\
& =\left\langle L M_{i-1}\right\rangle_{F}+\left\langle L \mathscr{L}_{i-1}\right\rangle_{F}=\left\langle L M_{i-1}\right\rangle_{F}+\mathscr{L}_{i} .
\end{aligned}
$$

Включение $\mathscr{L}_{i} \subseteq A(f)$ доказывается индукцией по $i$, а обратное включение следует из того, что $\bigcup_{i \geqslant 0} \mathscr{L}_{i}$ - левый идеал кольца $A$, содержащий элемент $f$. Ясно, что найдется такое натуральное число $i$, что $\mu_{i}=0$ или, равносильно, что $\mathscr{L}_{i}={ }_{A}(f)$.

Шаг 2. Строится базис $f_{1}^{(1)}, \ldots, f_{(1)}^{k_{1}}$ линейной оболочки $\mathscr{L}_{1}$ системы элементов $\{g f: g \in L\}$ над полем $\mathbf{F}_{q}$ такой, что матрица координат его элементов в стандартном базисе имеет специальный ступенчатый вид (ведущий элемент каждой строки равен 1 и является единственным ненулевым элементом соответствующего столбца). Каждый элемент, полученный на этом шаге, помечается как новый.

Шаг 3. Предположим, что базис пространства $\mathscr{L}_{i}$ уже построен и имеет вид

$$
e_{1}, \ldots, e_{s}, f_{i}^{(1)}, \ldots, f_{i}^{\left(k_{i}\right)}
$$

где $e_{1}, \ldots, e_{s}$ - базис пространства $\mathscr{L}_{i-1}$, а $f_{i}^{(1)}, \ldots, f_{i}^{\left(k_{i}\right)}-$ базис пространства $\mu_{i-1}$, $s=0$ при $i=1$ и $s=\sum_{j=1}^{i-1} k_{i}$ при $i>1$, причем матрица координат его элементов в стандартном базисе имеет специальный ступенчатый вид (возможно, с перестановкой строк) и элементы $f_{i}^{(1)}, \ldots, f_{i}^{\left(k_{i}\right)}$ помечены как новые. Каждый из новых элементов умножается слева на все элементы лупы (в результате возникает система образующих подпространства $\left.\left\langle L \mathscr{L}_{i-1}\right\rangle_{F}\right)$, и если произведение $f_{i+1}^{(j)}=g f_{i}^{(k)}$ не лежит в линейной оболочке уже найденных базисных векторов, то мы добавляем линейную комбинацию уже построенных векторов и этого произведения, меняя уже построенные элементы таким образом, чтобы специальный ступенчатый вид матрицы координат сохранялся. Точнее, пусть к подиространству $\mathscr{L}_{i}$ уже добавлены элементы $f_{i+1}^{(1)}, \ldots, f_{i+1}^{(j-1)}$, причем базис полученного подпространства имеет вид $e_{1}^{\prime}, \ldots, e_{t}^{\prime}$, где $t=s+k_{i}+j-1$, и

$$
\left\langle e_{1}^{\prime}, \ldots, e_{t}^{\prime}\right\rangle=\mathscr{L}_{i} \oplus\left\langle e_{s+k_{i}+1}^{\prime}, \ldots, e_{t}^{\prime}\right\rangle=\mathscr{L}_{i} \oplus\left\langle f_{i+1}^{(1)}, \ldots, f_{i+1}^{(j-1)}\right\rangle
$$


Добавление элемента $f_{i+1}^{(j)}$ сводится к следующим преобразованиям:

(a) Элемент $f_{i+1}^{(j)}$ заменяется на

$$
e_{t+1}^{\prime \prime}=\alpha\left(f_{i+1}^{(j)}+\sum_{k=1}^{t} \beta_{k} e_{k}^{\prime}\right.
$$

для некоторых $\alpha \in F \backslash\{0\}, \beta_{1}, \ldots, \beta_{t} \in F$;

(b) Элементы $e_{k}^{\prime}$ заменяются на элементы

$$
e_{k}^{\prime \prime}=e_{k}^{\prime}+\gamma_{k} e_{i+1}^{\prime \prime}, \quad \gamma_{k} \in F, \quad k=1, \ldots, t
$$

Коэффициенты $\alpha, \beta_{k}$ и $\gamma_{k}$ подбираются так, чтобы обеспечить специальный ступенчатый вид матрицы координат новых базисных элементов. Однако, независимо от выбора этих коэффициентов, имеет место соотношение, подобное (1):

$$
\begin{aligned}
\left\langle e_{1}^{\prime \prime}, \ldots, e_{t+1}^{\prime \prime}\right\rangle & =\left\langle e_{1}^{\prime}, \ldots, e_{t}^{\prime}\right\rangle \oplus\left\langle e_{t+1}^{\prime \prime}\right\rangle \\
& =\left\langle e_{1}^{\prime}, \ldots, e_{t}^{\prime}\right\rangle \oplus\left\langle f_{i+1}^{(j)}\right\rangle \\
& =\mathscr{L}_{i} \oplus\left\langle e_{s+k_{i}+1}^{\prime}, \ldots, e_{t}^{\prime}\right\rangle \oplus\left\langle f_{i+1}^{(j)}\right\rangle \\
& =\mathscr{L}_{i} \oplus\left\langle f_{i+1}^{(1)}, \ldots, f_{i+1}^{(j)}\right\rangle .
\end{aligned}
$$

Затем все элементы перестроенного базиса с номерами $k=1, \ldots, s$ помечаются как старые, а элементы, добавленные на данном шаге, как новые. Из построения ясно (см. (1) или (2)), что новые элементы образуют базис некоторого подпространства $\mu_{i}$ такого, что

$$
\mathscr{L}_{i+1}=\mathscr{L}_{i} \oplus \mu_{i}
$$

(согласно первому утверждению предложения 1).

Шаг 4. Шаг 3 повторяется до тех пор, пока некоторые новые элементы будут добавляться к базису (заметим, что для групповой алгебры число повторений шага 3 равно 1). В результате, согласно второму утверждению предложения 1 , будет получен базис левого идеала, порожденного заданным элементом. Обозначим через $k$ размерность полученного идеала.

Шаг 5. По списку уже построенных главных идеалов, упорядоченному по размерности, с помощью массива указателей на первый идеал заданной размерности, проверяется совпадение уже построенного идеала с каким-либо из найденных ранее идеалов размерности $k$ (достаточно проверять принадлежность образующей $f$ к главному идеалу той же размерности $k$ ). В случае совпадения переходим к следующему элементу луповой алгебры (см. шаг 1), если же рассматриваемый элемент не принадлежит ни одному из идеалов заданной размерности $k$, то идеал добавлется к списку (с корректировкой упомянутого выше массива указателей). После этого происходит переход к следующему элементу луповой алгебры). 
Основной шаг 2. Имея базис для каждого главного идеала, строим новые идеалы как суммы главных.

Шаг 6. Сначала, суммируя главные идеалы попарно и отбрасывая те, что совпадают с уже построенными (аналогично шагу 5), получаем список идеалов ранга 2 (под рангом мы понимаем здесь минимальное число образующих).

Шаг 7. Беря суммы уже построенных идеалов ранга $r$ и главных идеалов (список которых построен на основном шаге 1) и пропуская уже найденные, получаем список идеалов ранга $r+1$.

Шаг 8. Шаг 7 повторяется, пока число вновь построенных идеалов отлично от нуля.

\section{3. Параметры луповых кодов}

На следуюшем этапе работы мы сравнивали параметры, полученные для луповых кодов, с параметрами лучших кодов в различных смыслах. Для этого определялось соответствие параметров некоторым хорошо известным границам для кодов. Были вычислены параметры всевозможных кодов вида $\mathscr{K}=\mathscr{K}(I)$ для всех левых идеалов $I$ луповых алгебр $\mathbf{F}_{q} G$ маленьких порядков (для $q \in\{2,3,4,5\}$ и для всех луп $G$ порядков $4,5,6,7$ ) и найдены наилучшие среди таких кодов. Приведем некоторые из полученных результатов.

\section{1. Граница Синглтона. МДР-коды. Оптимальные коды}

Напомним, что линейный $[n, k, d]$-код над конечным полем $\mathbf{F}_{q}$ удовлетворяет неравенству

$$
d \leqslant n-k+1
$$

(граница Синглтона) [8]; он называется МДР-кодом, если достигается равенство

$$
d=n-k+1 \text {. }
$$

Легко видеть, что любая луповая алгебра

$$
A=\mathbf{F}_{q} L
$$

содержит два тривиальных луповых МДР-кода: $[n, 1, n]$-код $\mathscr{K}\left(I_{0}\right)$, соответствующий идеалу

$$
I_{0}=A\left(\sum_{g \in G} g\right)=\mathbf{F}_{q}\left(\sum_{g \in G} g\right)
$$

и $[n, n-1,2]$-код $\mathscr{K}(\Delta)$, соответствующий фундаментальному идеалу $\Delta \triangleleft A$, который можно определить как левый (и правый) аннулятор идеала $I_{0}$.

Однако некоторые из исследованных алгебр содержат нетривиальные луповые МДРкоды, например, алгебра $F_{5} S_{3}$ содержит $[6,3,4]$-код. Были найдены луповые МДР-коды с следующими параметрами, приведенными в таблице 1 .

Напомним, что (не обязательно линейный) $[n, k, d]$-код $\mathscr{K}$ над конечным полем $\mathbf{F}_{q}$ называется оптимальным [7], если $|\mathscr{K}|=q^{k}-$ максимально возможная мощность $n$-кода с расстоянием $d$. В соответствии с этим определением введем следующее определение. 
Таблица 1. Луповые $[n, k, d]$-МДР-коды в $\mathbf{F}_{q} L$

\begin{tabular}{|c|l|l|l|l|}
\hline$n$ & $k$ & $d$ & $\mathbf{F}_{q}$ & Лупы $L$ \\
\hline 4 & 2 & 3 & $\mathbf{F}_{4}$ & $\mathbf{Z}_{2} \oplus \mathbf{Z}_{2}$ \\
\hline 4 & 2 & 3 & $\mathbf{F}_{5}$ & $\mathbf{Z}_{4}$ \\
\hline 5 & 3 & 3 & $\mathbf{F}_{4}$ & $\mathbf{Z}_{5}$ \\
\hline 5 & 3 & 3 & $\mathbf{F}_{5}$ & $\mathbf{Z}_{5}$ \\
\hline 5 & 2 & 4 & $\mathbf{F}_{4}$ & $\mathbf{Z}_{5}$ \\
\hline 5 & 2 & 4 & $\mathbf{F}_{5}$ & $\mathbf{Z}_{5}$ \\
\hline 6 & 3 & 4 & $\mathbf{F}_{5}$ & $\mathbf{Z}_{6}, S_{3}$ \\
\hline
\end{tabular}

Определение 1. Линейный $[n, k, d]_{q}$-код называется линейно оптимальным, если $k-$ максимально возможная размерность линейного над полем $\mathbf{F}_{q}$ кода длины $\boldsymbol{n}$ с расстоянием $d$.

Очевидно, что любой МДР-код оптимален. Сверх того, можно указать следующий признак линейной оптимальности.

Обозначим через $n(k, q)$ (соответственно, $m(k, q)$ ) максимальную длину МДР-кода $\mathcal{K}$ комбинаторной размерности $k$ над алфавитом из $q$ элементов (соответственно, линейного МДР-кода над полем $\mathbf{F}_{q}$ для примарного числа $\left.q\right)$. Конечно,

$$
m(k, q) \leqslant n(k, q) .
$$

Предложение 2. Пусть $n, k$ - натуральные числа, $q$ - такое примарное число, что $n>m(k+1, q)$. Тогда любой линейный $[n, k, n-k]_{q}$-код линейно оптимален.

Действительно, в противном случае существует линейный $[n, k+1, n-k]_{q}$-код. Но такой код является МДР-кодом, следовательно, $n \leqslant m(k+1, q)$, получаем противоречие.

Наши результаты показывают, что среди луповых кодов имеются коды, удовлетворяющие условию предложения 2. Например, каждая из алгебр $\mathbf{F}_{q} \mathscr{D}_{4}, \mathbf{F}_{q} Q_{8}, q=2,3,4,5$, содержит $[8,4,4]_{q}$-код. Все эти коды линейно оптимальны, поскольку для указанных значений $q$ выполнено неравенство

$$
m(5, q)<8
$$

в силу следующего хорошо известного результата.

Теорема $1([7,8])$. Если $q \leqslant k$, то

$$
n(k, q)=k+1
$$

Если $k \leqslant q$ и q четно, то

$$
n(k, q) \leqslant q+k-1 .
$$

Еели $3 \leqslant k \leqslant q$ и q нечетно, то

$$
n(k, q) \leqslant q+k-2
$$

Были получены следующие результаты, приведенные в таблице 2 (таблицы Кэли лупы \#1 и лупы \#2 приведены ниже). 
Таблица 2. Линейно оптимальные луповые $[n, k, d]$-коды в $\mathbf{F}_{q} L$

\begin{tabular}{|l|l|l|l|l|}
\hline$n$ & $k$ & $d$ & $\mathbf{F}_{q}$ & Лупы $L$ \\
\hline 6 & 2 & 4 & $\mathbf{F}_{2}$ & $\mathbf{Z}_{6}, S_{3}$, Лупа \#1 и еще 10 неассоциативных луп \\
\hline 6 & 2 & 4 & $\mathbf{F}_{3}$ & $\mathbf{Z}_{6}, S_{3}$, Лупа \#1 и еще 10 неассоциативных луп \\
\hline 6 & 3 & 3 & $\mathbf{F}_{3}$ & $\mathbf{Z}_{6}, S_{3}$, Лупа \#2 и еще 3 неассоциативных лупы \\
\hline 6 & 3 & 3 & $\mathbf{F}_{4}$ & $\mathbf{Z}_{6}, S_{3}$, Лупа \#2 и еще 3 неассоциативных лупы \\
\hline 7 & 4 & 3 & $\mathbf{F}_{2}$ & $\mathbf{Z}_{7}$ \\
\hline 7 & 4 & 3 & $\mathbf{F}_{4}$ & $\mathbf{Z}_{7}$ \\
\hline 7 & 3 & 4 & $\mathbf{F}_{2}$ & $\mathbf{Z}_{7}$ \\
\hline 7 & 3 & 4 & $\mathbf{F}_{4}$ & $\mathbf{Z}_{7}$ \\
\hline
\end{tabular}

Таблища 3. Таблицы Кэли луп \#1, \#2

$$
\text { Лупа \#1: }
$$

Лупа \#2:

$\begin{array}{llllllllllll}0 & 1 & 2 & 3 & 4 & 5 & 0 & 1 & 2 & 3 & 4 & 5 \\ 1 & 0 & 3 & 2 & 5 & 4 & 1 & 0 & 3 & 2 & 5 & 4 \\ 2 & 3 & 4 & 5 & 0 & 1 & 2 & 3 & 4 & 5 & 0 & 1 \\ 3 & 2 & 5 & 4 & 1 & 0 & 3 & 4 & 5 & 0 & 1 & 2 \\ 4 & 5 & 0 & 1 & 3 & 2 & 4 & 5 & 0 & 1 & 2 & 3 \\ 5 & 4 & 1 & 0 & 2 & 3 & 5 & 2 & 1 & 4 & 3 & 0\end{array}$

\section{2. Граница Плоткина}

Для любого $[n, k, d]_{q}$-кода выполняется неравенство (граница Плоткина [8])

$$
d \leqslant \frac{(q-1) q^{k}}{q\left(q^{k}-1\right)} n .
$$

Если оно обращается в равенство, то мы говорим, что данный код лежит на границе Плоткина.

Таблища 4 показывает, какие луповые коды лежат на границе Плоткина.

\section{3. Граница Варшамова-Гилберта}

Пусть

$$
s_{q}(i, \delta)=\sum_{0 \leqslant t \leqslant \delta}\left(\begin{array}{l}
i \\
t
\end{array}\right)(q-1)^{t}
$$

(число элементов шара радиуса $\delta$ в пространстве Хемминга $F_{q}^{i}$ ). Используя 1.10 из [8] и 8.6 из [7], нетрудно доказать следующий факт.

Таблица 4. Луповые $[n, k, d]$-коды в $\mathbf{F}_{q} L$, лежащие на границе Плоткина

\begin{tabular}{|l|l|l|l|l|}
\hline$n$ & $k$ & $d$ & $\mathbf{F}_{q}$ & Лупы $L$ \\
\hline 5 & 2 & 4 & $\mathbf{F}_{4}$ & $\mathbf{Z}_{5}$ \\
\hline 6 & 2 & 4 & $\mathbf{F}_{2}$ & $\mathbf{Z}_{6}, S_{3}$, Лупа \#1 и еще 10 неассоциативных луп \\
\hline 7 & 3 & 4 & $\mathbf{F}_{2}$ & $\mathbf{Z}_{7}$ \\
\hline
\end{tabular}


Таблица 5. Луповые $[n, k, d]$-коды в $\mathbf{F}_{q} L$, удовлетворяющие условию Варшамова-Гилберта

\begin{tabular}{|c|c|c|l|l|}
\hline$n$ & $k$ & $d$ & $\mathbf{F}_{q}$ & Лупы $L$ \\
\hline 4 & 2 & 3 & $\mathbf{F}_{4}$ & $\mathbf{Z}_{2} \oplus \mathbf{Z}_{2}$ \\
\hline 4 & 2 & 3 & $\mathbf{F}_{5}$ & $\mathbf{Z}_{4}$ \\
\hline 5 & 3 & 3 & $\mathbf{F}_{4}$ & $\mathbf{Z}_{5}$ \\
\hline 5 & 3 & 3 & $\mathbf{F}_{5}$ & $\mathbf{Z}_{5}$ \\
\hline 5 & 2 & 4 & $\mathbf{F}_{4}$ & $\mathbf{Z}_{5}$ \\
\hline 5 & 2 & 4 & $\mathbf{F}_{5}$ & $\mathbf{Z}_{5}$ \\
\hline 6 & 3 & 4 & $\mathbf{F}_{5}$ & $\mathbf{Z}_{6}, S_{3}$ \\
\hline
\end{tabular}

Теорема 2. Для заданных чисел $n \in \mathbf{N}, d \in\{1, \ldots, n-1\}$ существует линейный $[n, k, d]_{q^{-}}$ код такой, что

$$
k \geqslant n-\left[\log _{q} s_{q}(n-1, d-2)\right]-1 .
$$

Если линейный $[n, k, d]_{q}$-код удовлетворяет неравенству из этой теоремы, мы говорим, что он удовлетворяет условию Варшамова-Гилберта.

Вычисления показывают, что существуют луповые коды, удовлетворяющие условию Варшамова-Гилберта.

\section{4. Параметры групповых кодов}

Поскольку число луп порядка $n$ растет очень быстро при возрастании $n$, в случае луповых кодов длины, большей 7, мы ограничились рассмотрением групповых кодов. Изучались параметры групповых кодов для следующих групп: групп диэдра $\mathscr{D}_{m}, m=4,5,6$; группы кватернионов $Q_{8}$; знакопеременной группы $A_{4}$ и всех абелевых групп порядков 8 , 9, 10 и 12 (коды из групповых алгебр $\mathbf{F}_{q} \mathbf{Z}_{11}$ не включены в таблицы, поскольку для рассматриваемых значений $q$ это - известные классические циклические коды [8]).

Приведем список линейно оптимальных кодов, которые лежат в групповых алгебрах указанных групп и удовлетворяют предложению 2 (таблица 6), а также список найденных групповых кодов, лежащих на гранище Плоткина.

\section{5. Заключение}

1. Решетки левых идеалов неассоциативных луповых алгебр $A=\mathrm{F}_{q} L$ малой мощности $(q \leqslant 5,|L| \leqslant 7)$, за редким исключением, тривиальны и состоят из следующих четырех идеалов: $0, I_{0}, \Delta, A$. Поэтому они не содержат интересных луповых кодов.

Исключение составляют линейно оптимальные $[6,2,4]_{q}$-коды для $q \in\{2,3\}$, а также $[6,3,3]_{q}$-коды для $q \in\{3,4\}$. Отметим, что найденные $[6,2,4]_{q}$-коды не только линейно оптимальны, но также абсолютно оптимальны (см. [8]).

Представляется естественной возможность построения более интересных луповых кодов для луп больших порядков, однако полный перебор всех таких кодов - более сложная задача.

2. При изучении перечисленных выше групповых кодов были найдены, в частности, следующие известные абсолютно оптимальные (в соответствие с [8]) линейные коды: 
Таблица 6. Линейно оптимальные групповые $[n, k, d]$-коды в $\mathrm{F}_{q} G$

\begin{tabular}{|c|c|c|l|l|}
\hline$n$ & $k$ & $d$ & $\mathbf{F}_{q}$ & Группы $G$ \\
\hline 8 & 4 & 4 & $\mathbf{F}_{2}$ & $\mathbf{Z}_{2} \oplus \mathbf{Z}_{2} \oplus \mathbf{Z}_{2}, \mathbf{Z}_{4} \oplus \mathbf{Z}_{2}, Q_{8}, \mathscr{D}_{4}$ \\
\hline 8 & 5 & 3 & $\mathbf{F}_{3}$ & $\mathbf{Z}_{8}, Q_{8}$ \\
\hline 8 & 4 & 4 & $\mathbf{F}_{3}$ & $\mathbf{Z}_{2} \oplus \mathbf{Z}_{2} \oplus \mathbf{Z}_{2}, \mathbf{Z}_{4} \oplus \mathbf{Z}_{2}, \mathbf{Z}_{8}, Q_{8}, \mathscr{D}_{4}$ \\
\hline 8 & 3 & 5 & $\mathbf{F}_{3}$ & $\mathbf{Z}_{8}, Q_{8}$ \\
\hline 8 & 2 & 6 & $\mathbf{F}_{3}$ & $\mathbf{Z}_{8}, Q_{8}$ \\
\hline 8 & 5 & 3 & $\mathbf{F}_{4}$ & $\mathbf{Z}_{2} \oplus \mathbf{Z}_{2} \oplus \mathbf{Z}_{2}, \mathbf{Z}_{4} \oplus \mathbf{Z}_{2}, Q_{8}, \mathscr{D}_{4}$ \\
\hline 8 & 4 & 4 & $\mathbf{F}_{4}$ & $\mathbf{Z}_{2} \oplus \mathbf{Z}_{2} \oplus \mathbf{Z}_{2}, \mathbf{Z}_{4} \oplus \mathbf{Z}_{2}, Q_{8}, \mathscr{D}_{4}$ \\
\hline 8 & 2 & 6 & $\mathbf{F}_{4}$ & $\mathbf{Z}_{2} \oplus \mathbf{Z}_{2} \oplus \mathbf{Z}_{2}, \mathbf{Z}_{4} \oplus \mathbf{Z}_{2}, Q_{8}, \mathscr{D}_{4}$ \\
\hline 8 & 5 & 3 & $\mathbf{F}_{5}$ & $\mathbf{Z}_{4} \oplus \mathbf{Z}_{2}, \mathbf{Z}_{8}, Q_{8}, \mathscr{D}_{4}$ \\
\hline 8 & 4 & 4 & $\mathbf{F}_{5}$ & $\mathbf{Z}_{2} \oplus \mathbf{Z}_{2} \oplus \mathbf{Z}_{2}, \mathbf{Z}_{8}, Q_{8}, \mathscr{D}_{4}$ \\
\hline 8 & 2 & 6 & $\mathbf{F}_{5}$ & $\mathbf{Z}_{4} \oplus \mathbf{Z}_{2}, \mathbf{Z}_{8}$ \\
\hline 8 & 3 & 5 & $\mathbf{F}_{4}$ & $Q_{8}$ \\
\hline 9 & 6 & 3 & $\mathbf{F}_{3}$ & $\mathbf{Z}_{3} \oplus \mathbf{Z}_{3}$ \\
\hline 9 & 5 & 4 & $\mathbf{F}_{3}$ & $\mathbf{Z}_{3} \oplus \mathbf{Z}_{3}$ \\
\hline 9 & 4 & 5 & $\mathbf{F}_{3}$ & $\mathbf{Z}_{3} \oplus \mathbf{Z}_{3}$ \\
\hline 9 & 3 & 6 & $\mathbf{F}_{3}$ & $\mathbf{Z}_{3} \oplus \mathbf{Z}_{3}$ \\
\hline 9 & 6 & 3 & $\mathbf{F}_{4}$ & $\mathbf{Z}_{3} \oplus \mathbf{Z}_{3}$ \\
\hline 9 & 3 & 6 & $\mathbf{F}_{4}$ & $\mathbf{Z}_{3} \oplus \mathbf{Z}_{3}$ \\
\hline 10 & 6 & 4 & $\mathbf{F}_{4}$ & $\mathbf{Z}_{10}, \mathscr{D}_{5}$ \\
\hline 10 & 2 & 8 & $\mathbf{F}_{4}$ & $\mathbf{Z}_{10}, \mathscr{D}_{5}$ \\
\hline 10 & 7 & 3 & $\mathbf{F}_{5}$ & $\mathbf{Z}_{10}, \mathscr{D}_{5}$ \\
\hline 10 & 6 & 4 & $\mathbf{F}_{5}$ & $\mathbf{Z}_{10}, \mathscr{D}_{5}$ \\
\hline 10 & 2 & 8 & $\mathbf{F}_{5}$ & $\mathbf{Z}_{10}, \mathscr{D} 5$ \\
\hline 10 & 4 & 6 & $\mathbf{F}_{4}$ & $\mathscr{D}_{5}$ \\
\hline 10 & 4 & 6 & $\mathbf{F}_{5}$ & $\mathscr{D}_{5}$ \\
\hline 12 & 9 & 3 & $\mathbf{F}_{4}$ & $\mathbf{Z}_{2} \oplus \mathbf{Z}_{6}, A_{4}$ \\
\hline 12 & 9 & 3 & $\mathbf{F}_{5}$ & $\mathbf{Z}_{2} \oplus \mathbf{Z}_{6}, A_{4}$ \\
\hline 12 & 8 & 4 & $\mathbf{F}_{5}$ & $\mathbf{Z}_{2} \oplus \mathbf{Z}_{6}, \mathbf{Z}_{2} \oplus \mathbf{Z}_{6} . A_{4}, \mathscr{D}_{6}$ \\
\hline 12 & 8 & 4 & $\mathbf{F}_{4}$ & $A_{4}$ \\
\hline 12 & 6 & 6 & $\mathbf{F}_{4}$ & $A_{4}$ \\
\hline 12 & 6 & 6 & $\mathbf{F}_{4}$ & $\mathscr{D}_{6}$ \\
\hline
\end{tabular}

Таблица 7. Групповые $[n, k, d]$-коды в $\mathrm{F}_{q} G$, лежащие на границе Плоткина

\begin{tabular}{|c|c|c|c|l|}
\hline$n$ & $k$ & $d$ & $\mathbf{F}_{q}$ & Группы $G$ \\
\hline 8 & 2 & 6 & $\mathbf{F}_{3}$ & $\mathbf{Z}_{8}, Q_{8}$ \\
\hline 9 & 2 & 6 & $\mathbf{F}_{2}$ & $\mathbf{Z}_{3} \oplus \mathbf{Z}_{3}, \mathbf{Z}_{9}$ \\
\hline 10 & 2 & 8 & $\mathbf{F}_{4}$ & $\mathbf{Z}_{10}, \mathscr{D}_{5}$ \\
\hline 12 & 2 & 8 & $\mathbf{F}_{2}$ & $\mathbf{Z}_{2} \oplus \mathbf{Z}_{6}, \mathbf{Z}_{1} 2, A_{4}, \mathscr{D}_{6}$ \\
\hline
\end{tabular}


симплексный $[7,3,4]_{2}$-код в $\mathbf{F}_{2} \mathbf{Z}_{7} ;[7,4,3]_{2}$-код Хэмминга в $\mathbf{F}_{2} \mathbf{Z}_{7} ;[8,4,4]_{2}$-код Бауэра [7] (расширенный $[7,4,3]_{2}$-код Хэмминга) в $\mathbf{F}_{2}\left(\mathbf{Z}_{2}^{3}\right), \mathbf{F}_{2}\left(\mathbf{Z}_{2} \times \mathbf{Z}_{4}\right), \mathbf{F}_{2} Q_{8}, \mathbf{F}_{2} D_{4}$, где $Q_{8}-$ группа кватернионов, $D_{4}$ - группа диэдра.

3. В групповых алгебрах некоммутативных групा существуют линейно оптимальные коды, которые нельзя построить с использованием абелевых групп. Примеры таких кодов: $[8,3,5]$-код в $\mathbf{F}_{4} Q_{4} ;[10,4,6]$-код в $\mathbf{F}_{4} D_{5}$ и в $\mathbf{F}_{5} D_{5} ;[12,8,4]$ - и $[12,6,6]$-коды в $\mathbf{F}_{4} A_{4}$; $[12,6,6]$-код в $\mathbf{F}_{4} D_{6}$.

4. Важно отметить, что многие из найденных нами лучших кодов задаются идеалами из не полупростых и некоммутативных алгебр. Это делает особо интересной задачу изучения таких алгебр с точки зрения теории кодов.

5. В $[6,7]$ было показано, что если $G$ - циклическая группа порядка $q+1$ и $k=1, \ldots, q$, то алгебра $\mathrm{F}_{q} G$ содержит идеал $I$ такой, что $\mathscr{K}(I)$ есть $[q+1, k, q-k+2]-(\mathrm{MДР)-код,} \mathrm{если} \mathrm{и}$ только если $q$ четно или $k$ нечетно. Кроме того, в [5] было показано, что если $q$ нечетно и $k$ четно, то существует не циклический, но рекурсивный над $\mathbf{F}_{q}[q+1, k, q-k+2]$-(МДР)код. Интересно, существуют ли такие луповые коды для каких-нибудь нециклических луп $L$ ? Наши вычисления показывают, что для $q=3,5$ и всех подходящих четных $k$ таких кодов не существует. Более того, не существует групповых кодов с указанными параметрами для $q=7,9$ и соответствующих четных $k$. Это позволяет предположить, что верна следующая гипотеза.

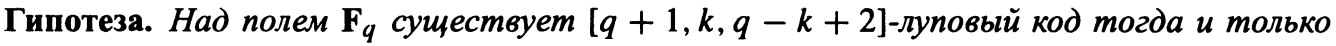
тогда, когда q четно или $k$ нечетно, то есть тогда и только тогда, когда существует циклический код с указанными параметрами.

6. Для $q=2^{t}$ существует линейный $[q+2, q-1,4]-\left(\mathrm{MДP)-код} \mathrm{над} \mathbf{F}_{q}-\right.$ дважды расширенный код Рида-Соломона (см., например, [8]). Линейный над $\mathbf{F}_{q}$ циклический код с такими параметрами неизвестен, однако в [5] было показано, что один из таких кодов линейно (в широком смысле) эквивалентен циклическому линейному над пространством $\mathbf{F}_{2}^{t}$ коду, названному Астурийским кодом. В связи с этим было бы интересно найти лупу $L$ порядка $q+2$ такую, что алгебра $\mathbf{F}_{q} L$ содержит идеал, задающий $[q+2, q-1,4]$-код.

Тот же вопрос интересен относительно $[q+2,3, q]$-кода, двойственного к исходному.

\section{Список литературы}

1. Абашин А. С., Линейные рекурсивные МДР-коды размерности 2 и 3. Дискретная математика (2000) 12, №2, 140-153.

2. Гонсалес С., Коусело Е., Марков В. Т., Нечаев А. А., Рекурсивные МДР-коды и рекурсивно дифференцируемые квазигруппы. Дискретная математика (1998) 10, №2, 3-29.

3. Couselo E., Gonzalez S., Markov V., Nechaev A., Recursive MDS-codes and recursively differenti-able $k$-quasigroups. Proc. Sixth Intern. Workshop on Algebraic and Combinatorial Coding Theory. Pskov, 1998, 78-84.

4. Couselo E., Gonzalez S., Markov V., Nechaev A., Recursive MDS-codes. Proc. WCC'99. Paris, 1999, 271-278. 
5. Couselo E., Gonzalez S., Markov V., Nechaev A., Linear recursive MDS-codes and Asturian codes. Proc. WCC'01. Paris, 2001, 149-156.

6. Georgiades J., Cyclic $(q+1, k)$-codes of odd order $q$ and even dimension $k$ are not optimal. Atti Sem. Mat. Fis. Univ. Modena (1982) 30, 284-285.

7. Heise W., Quattrocci P., Informations- und Codierungstheorie. Springer, Berlin, 1995.

8. МакВилльямс Ф. Дж., Слоэн Н. Дж. А., Теория кодов, исправляющих ошибки. Связь, Москва, 1979.

9. Нечаев А. А., Кузьмин А. С., Марков В. Т., Линейные коды над конечными кольцами и модулями. Фундаментальная и прикладная математика (1997) 3, №1, 195-254.

10. Chein O., Pflugfelder H. O., Smith J. D. H., Eds., Quasigroups and loops: theory and applications. Heldermann, Berlin, 1990.

11. Sabin R. E., Lomonaco S. J., Metacyclic error-correcting codes. Appl. Algebra Eng. Commun. Comput. (1995) 6, №3, 191-210.

12. Sabin R. E., On determining all codes in semi-simple group rings. Lect. Notes Comput. Sci. (1993) 673, 279-290.

13. Sabin R. E., An ideal structure for some quasi-cyclic error-correcting codes. Lect. Notes Pure Appl. Math. (1993) 141, 183-194.

Статья поступила 10.11.2003. 